\title{
Pneumopathie nécrosante à Staphylococcus aureus
}

\section{Necrotizing pneumonia due to Staphylococcus aureus}

\author{
M. Dujardin · E. Beti · C. Vanhecke \\ Reçu le 11 janvier 2018; accepté le 21 mars 2018 \\ (C) SFMU et Lavoisier SAS 2018
}

\section{Introduction}

Lors de la saison grippale à La Réunion, département d'outre-mer situé dans l'océan Indien, les surinfections pulmonaires sont un motif fréquent de consultation. Par contre, les pneumopathies nécrosantes à staphylocoques sont beaucoup plus rares. La gravité potentielle de cette infection oblige à une attention particulière dans l'approche diagnostique et thérapeutique dès l'admission du patient dans le service des urgences.

\section{Observation}

Un patient de 43 ans s'est présenté aux urgences pour un syndrome grippal évoluant depuis six jours. Dans ses antécédents, on notait une hépatite $\mathrm{C}$ ancienne. Il n'y avait pas de voyage récent à l'étranger relaté. L'examen clinique était rassurant, sans signe de lutte, et l'hémodynamique était conservée. Les bilans biologiques et radiographiques étaient en faveur d'une pneumopathie franche lobaire aiguë droite communautaire. Devant l'absence de signe de gravité, de comorbidité et le bon état général, le patient est traité en ambulatoire.

Après deux jours de traitement, le patient consulte à nouveau aux urgences pour une dégradation de son état clinique associant une hémoptysie et une douleur thoracique droite. L'examen clinique retrouvait alors des signes de détresse respiratoire. L'auscultation pulmonaire notait des crépitants dans le champ pulmonaire droit avec une diminution du murmure vésiculaire. L'hémodynamique notifiait une pres-

M. Dujardin · E. Beti $\cdot$ C. Vanhecke $(\bowtie)$

Service des urgences-Smur, centre hospitalier Gabriel-Martin,

38, rue Labourdonnais CS 11045, F-97166 Saint-Paul,

La Réunion

e-mail : christophevanhecke@yahoo.fr

C. Vanhecke

Centre René-Labusquière, institut de médecine tropicale, université Victor-Segalen, F-33000 Bordeaux, France sion artérielle à 126/90 mmHg, une tachycardie à 118/min, une saturation en air ambiant à $91 \%$ et une température à $36,1^{\circ} \mathrm{C}$. Le syndrome inflammatoire était majoré, avec une hyperleucocytose prédominante pour les polynucléaires neutrophiles $\left(14400 / \mathrm{mm}^{3}\right)$, une protéine $C$ réactive $(\mathrm{CRP})$ à $370 \mathrm{mg} / \mathrm{l}$ et une procalcitonine à $51,6 \mathrm{ng} / \mathrm{ml}$. La gazométrie en air ambiant montrait une hypoxémie à $60 \mathrm{mmHg}$, une $\mathrm{PaCO}_{2}$ à $32 \mathrm{mmHg}$ et un $\mathrm{pH}$ alcalin à 7,52. Le patient a bénéficié d'un examen scannographique pulmonaire qui objectivait une pneumopathie lobaire supérieure droite d'allure nécrosante (Fig. 1). Une antibiothérapie probabiliste par céfotaxime, spiramycine et gentamicine a été initiée aux urgences avant le transfert du patient dans le service de réanimation.

Lors de son séjour en réanimation, l'antibiothérapie a été modifiée par l'association de cloxacilline $2 \mathrm{~g} / 8 \mathrm{~h}$ et de clindamycine $600 \mathrm{mg} / 8 \mathrm{~h}$. En effet, les hémocultures et l'examen cytobactériologique des crachats sont revenus positifs à Staphylococcus aureus (S. aureus). L'évolution favorable après trois jours a permis un retour en service de médecine conventionnelle. Le résultat microbiologique du Centre national de référence confirmait l'infection à $S$. aureus méticillinosensible sécréteur de la leucocidine de Panton-Valentine (PVL+).

\section{Discussion}

S. aureus est un agent pathogène isolé dans 2 à $5 \%$ des pneumopathies communautaires, plus fréquemment rencontré chez le sujet âgé avec des comorbidités, dans un contexte grippal. Toutefois, S. aureus peut être parfois sécréteur d'une leucotoxine particulière, la $\mathrm{PVL}+$, responsable de pneumopathies nécrosantes graves, grevées d'une mortalité élevée entre 30 et $75 \%$ [1]. Les pneumopathies nécrosantes à $S$. aureus $\mathrm{PVL}+$ constituent des tableaux cliniques touchant majoritairement les enfants et les adultes jeunes, le plus souvent indemnes de comorbidité et dans un contexte de syndrome grippal. À la phase d'état, la présentation clinique, décrite par Gillet et al., est caractérisée par l'association d'une fièvre élevée $\left(>39^{\circ} \mathrm{C}\right)$, des infiltrations alvéolaires 

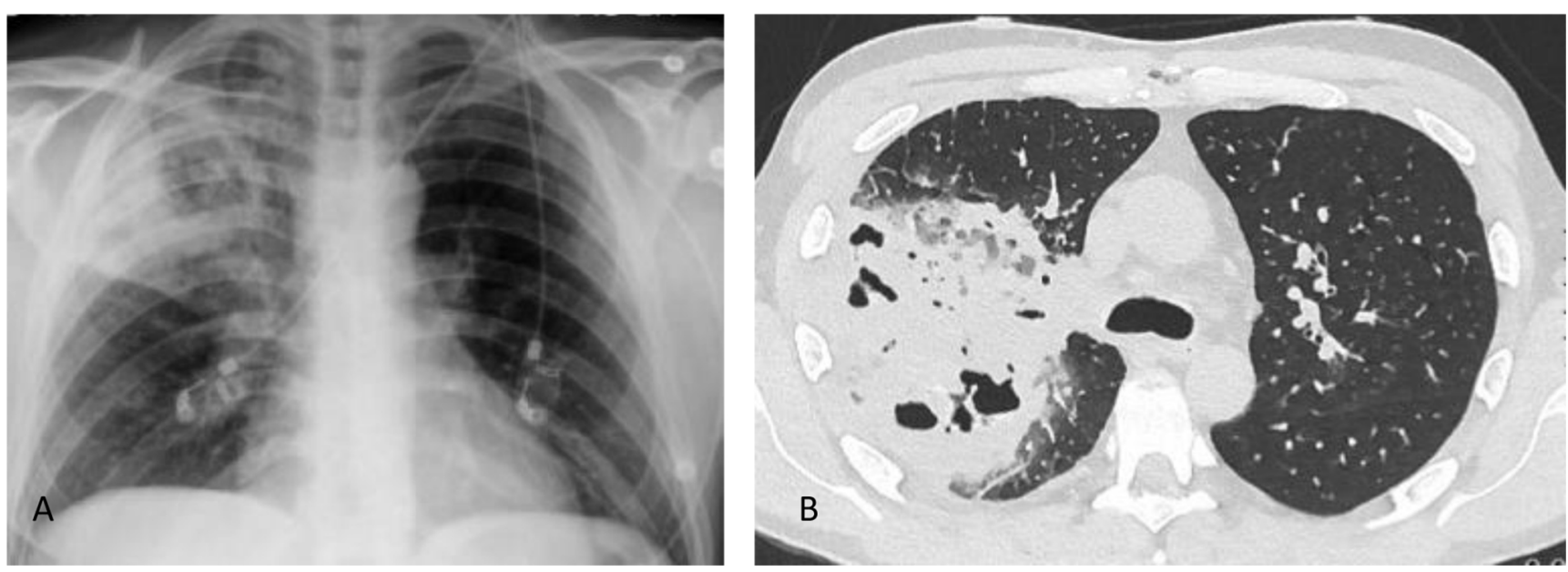

Fig. 1 A. Radiographie pulmonaire du patient à son admission, retrouvant une pneumopathie lobaire supérieure droite. B. Scanner thoracique : pneumopathie lobaire droite d'allure nécrosante

multilobaires, d'hémoptysies et d'une leucopénie [1]. Ce tableau est associé fréquemment à une détresse respiratoire rapide et à une instabilité hémodynamique. Dans $10 \%$ des cas, une érythrodermie généralisée est notifiée. Radiologiquement, il existe le plus souvent des infiltrats alvéolaires bilatéraux, multilobaires, généralement associés à des épanchements pleuraux (50\%). La biologie retrouve en général, une leucopénie basse $\left(<3000\right.$ leucocytes $\left./ \mathrm{mm}^{3}\right)$, avec un syndrome inflammatoire marqué et une CRP très élevée (>300 mg/l) (Tableau 1) [1]. Si ce tableau clinique classique semble facilement identifiable, des présentations atypiques ou incomplètes sont relativement fréquentes, rendant le diagnostic plus laborieux. En effet, dans l'observation que nous décrivons, même s'il existait un contexte grippal, le patient ne présentait pas de détresse respiratoire ni de leucopénie ou d'hémoptysie lors de la première consultation. Également, la radiographie initiale évoquait l'aspect d'une pneumopathie communautaire unilobaire. C'est devant l'évolution défavorable après 48 heures avec l'apparition secondaire d'hémoptysie et des images de nécrose au scanner que le diagnostic était évoqué.

Cette infection pulmonaire bactérienne à $S$. aureus $\mathrm{PVL}+$ est encore peu fréquente en France et dans le département ultramarin de l'océan Indien et est relativement méconnue des praticiens. Par conséquent, cela entraîne, le plus souvent, un retard dans le diagnostic, car rarement évoqué à l'admission. Il n'existe pas aujourd'hui de traitement standardisé dans la prise en charge de la pneumopathie nécrosante à S. aureus $\mathrm{PVL}+$. Le caractère très rapide de l'évolution souvent sombre de cette infection pose le problème de l'antibiothérapie probabiliste en cas de suspicion dès le service des urgences [2]. Le choix de l'antibiotique sera établi par l'association d'une molécule bactéricide avec une bonne diffusion pulmonaire active sur Staphylococcus, Streptococcus pneumoniae et Streptococcus pyogènes, et d'un anti-
Tableau 1 Présentation clinique classique d'une pneumopathie nécrosante à Staphylococcus aureus sécréteur de leucocidine de Panton-Valentine (PVL+)

Terrain
Enfant ou adulte jeune
Épisode grippal récent
Signes cliniques
Dyspnée
Hyperthermie $>39{ }^{\circ} \mathrm{C}$
Hémoptysie $^{\mathrm{a}}$
Érythrodermie $^{\mathrm{a}}$
SDRA $^{\mathrm{a}}$
Biologie
Leucopénie initiale basse (leucocytes $\left.<3000 / \mathrm{mm}^{3}\right)^{\mathrm{a}}$
Protéine C réactive très élevée $(>300 \mathrm{mg} / \mathrm{l})$
Radiographie pulmonaire
Infiltrats alvéolaires multilobaires ${ }^{\mathrm{a}}$
Épanchement pleural
Tomodensitométrie pulmonaire
Infiltrations multilobaires du parenchyme pulmonaire
avec cavités de taille variable
Évolution
Rapidement défavorable (SDRA, choc septique)
Taux de mortalité importante (environ $50 \%$ )
SDRA : syndrome de détresse respiratoire aiguë
${ }^{\text {a }}$ Critères de gravité et facteurs associés à la mortalité selon Gil-
let et al. [1]

infectieux réduisant la production de toxines, comme le linézolide, la clindamycine ou la rifampicine [3].

Devant l'émergence des souches de staphylocoques résistantes à la méticilline (SARM), les recommandations françaises actuelles dans la prise en charge des pneumopathies 
nécrosantes gravissimes préconisent une triple antibiothérapie probabiliste associant un glycopeptide, une céphalosporine de troisième génération et la clindamycine ou la rifampicine [4]. Cependant à La Réunion, la présence de SARM est encore exceptionnelle et ne nous invite pas à introduire la vancomycine en probabiliste, sauf en cas de retour d'une zone à forte prévalence de SARM (Afrique de l'Est, Comores, Madagascar, Asie), voyages de plus en plus fréquents à partir de l'île de La Réunion. Dans notre description, la clindamycine a été instaurée dès l'obtention des résultats des hémocultures à cocci Gram+ permettant une évolution favorable, malgré l'apparition d'une détresse respiratoire et d'hémoptysie, signes considérés de mauvais pronostic. En pratique, en cas de pneumopathie sévère du sujet jeune, dans un contexte postgrippal à La Réunion, il est nécessaire de prendre en compte la possibilité d'un éventuel $S$. aureus PVL+ pour le traitement empirique en plus des pathogènes habituels. Dans ce cas, et en l'absence de contre-indication, l'antibiothérapie probabiliste chez l'adulte pourrait être composée obligatoirement d'une céphalosporine de troisième génération, combinée à la clindamycine ou au linézolide, dès le service des urgences, avant l'adaptation au vu des résultats microbiologiques. La durée du traitement n'est pas consensuelle et reste controversée, variant de trois à huit semaines selon les formes cliniques [2]. En période épidémique grippale, un traitement antiviral peut être proposé. Parmi les thérapeutiques adjuvantes proposées, les immunoglobulines intraveineuses auraient leur place en cas de choc toxique à staphylocoque [5]. Leur utilisation précoce limiterait, en théorie, l'aggravation des lésions parenchymateuses.
En conclusion, la pneumopathie nécrosante à $S$. aureus PVL + est encore rare en France et à La Réunion, mais on connaît un accroissement des descriptions depuis quelques années. L'évolution rapide vers une détresse respiratoire aiguë oblige le médecin urgentiste, constamment en première ligne, à connaître et évoquer le diagnostic ainsi qu'une prise en charge optimale et précoce de cette pathologie mettant en jeu rapidement le pronostic vital.

Conflits d'intérêts : les auteurs déclarent ne pas avoir de lien d'intérêt.

\section{Références}

1. Gillet Y, Vanhems P, Lina G, et al (2007) Factors predicting mortality in necrotizing community-acquired pneumonia caused by Staphylococcus aureus containing Panton-Valentine leukocidin. Clin Infect Dis 45:315-21

2. Brulé N, Jaffré $S$, Chollet $S$, et al (2008) Pneumonie nécrosante à Staphylococcus aureus sécréteur de la toxine de Panton-Valentine. Rev Mal Respir 25:875-9

3. Escudier E, Pagani L, Gaillat J, et al (2016) Discussion autour d'un cas mortel de pneumonie nécrosante à Staphylococcus aureus sécrétant la leucocidine de Panton-Valentine. Réanimation 25:361-5

4. Gillet Y, Dumitrescu O, Tristan A, et al (2011) Pragmatic management of Panton-Valentine leukocidins associated staphylococcal diseases. Int J Antimicrob Agents 38:457-64

5. Morgan MS (2007) Diagnosis and treatment of Panton-Valentine leukocidin (PVL) associated staphylococcal pneumonia. Int Antimicrob Agents 30:289-96 